\title{
CURVA DE ABSORÇÃO DE ÁGUA EM SEMENTES DE ATEMÓIA (Annona cherimola MILL. x Annona squamosa L.) CV. GEFNER ${ }^{1}$
}

\author{
GISELA FERREIRA², VANDEIR FRANCISCO GUIMARÃES ${ }^{3}$, SHEILA ZAMBELLO DE PINHO ${ }^{4}$, MARCOS CAMPOS \\ DE OLIVEIRA $^{5}$, ALFREDO RICHART ${ }^{5}$, JOÃO FILGUEIRAS BRAGA ${ }^{6}$, GLÁUCIA BRAVO DIAS ${ }^{7}$
}

\begin{abstract}
RESUMO - O presente trabalho teve o objetivo de caracterizar a curva de absorção de água em sementes de atemóia (Annona cherimola Mill x Annona squamosa L.) cv. Gefner, submetidas a três métodos de embebição: sementes submersas em água destilada (MSSA), sementes entre papel de filtro embebido em água destilada acondicionada em caixa tipo gerbox (MPEA) e teste-padrão (MTP), com sementes mantidas em rolo de papel de filtro umedecido em água destilada. O delineamento experimental foi o inteiramente casualizado, com 6 tratamentos e 4 repetições de 25 sementes por parcela, constituídos por três métodos de embebição, empregando-se sementes vivas e mortas. O tempo de embebição entre 27; 34 e 47 horas, nos métodos MTP, MPEA e MSSA, representam indicativo para tratamento de sementes, podendo funcionar como tempo mínimo necessário para embebição em solução com reguladores vegetais. Conclui-se que os métodos que caracterizaram as três fases de absorção de água em sementes de atemóia foram o MTP e MPEA com mudança entre as fases I e II após 27 e 34 horas, respectivamente, atingindo a fase III com 234 horas, o que permite determinar o tempo de imersão para tratamentos pré-germinativos.
\end{abstract}

Termos para indexação: Annonaceae, fases da germinação, métodos para embebição.

\section{WATER ABSORPTION CURVE IN SEEDS OF ATEMOYA (Annona cherimola MILL. x Annona squamosa L.) CV.} GEFNER

\begin{abstract}
The present study aimed to characterize the water absorption curve in atemoya seeds (Annona cherimola Mill x Annona squamosa L.) cv. Gefner, submitted to three imbibitions methods: seeds submerged in distilled water (MSSA), seed placed betwen paper filter imbibed with distilled water inside a gerbox box (MPEA) and standard test, with seeds kept in roll of filter paper moistened with water (MTP). The experimental delineation was entirely randomized, with 6 treatments and 4 repetitions of 25 seeds per parcel. The treatments were submitted to three imbibitions methods, with live and deth seeds. The imbibition time among 27, 34 and 47 hours, in the MTP, MPEA and MSSA methods represented an indicative for seeds treatment, which could possibly function as a minimal time needed for imbibition in solution with vegetal regulators. The MTP and MPEA were the methodologies that provided a better curve characterization, with changes between phases I and II after 27 and 34 hours, and reaching the phase III after 234 hours, which suggests the immersion time for seeds treatments.
\end{abstract}

Index terms: Annonaceae, germination phases, imbibition methods.

A atemóia é um híbrido interespecífico entre Annona cherimola Mill. e Annona squamosa L. (Sanewski, 1991; Cereda \& Ferreira, 1997), sendo a enxertia o método usual para propagação (Sampaio, 1992). O emprego de sementes destina-se ao melhoramento genético e à produção de porta-enxertos (Sanewski, 1991; Cereda \& Ferreira, 1997) para Annona squamosa L. (Bourke, 1985) bem como para a própria atemóia (Sanewski, 1991; Stenzel, 1997).

Dentre os problemas do uso de sementes de atemóia para produção de porta-enxertos, destacam-se a heterogeneidade das mudas formadas e a baixa percentagem de germinação (Kavati et al., 1997; Stenzel, 1997) afetada pela dormência imposta com a presença de substâncias inibidoras e resistência do tegumento (Pawshe et al.,1997; Smet et al.,1999).

A germinação das sementes inicia-se com a embebição de água e desencadeia seqüência de mudanças metabólicas que culminam com a emergência de raiz primária, quando se refere a sementes viáveis nãodormentes (Laboriau, 1983; Bradford, 1995; Carvalho \& Nakagawa, 2000; Eira \& Caldas, 2000).

Bewley \& Black (1994) consideram que a absorção de água pelas sementes ocorre de acordo com padrão trifásico. A fase I, denominada embebição, é conseqüência de potencial matricial e, portanto, processo físico, que ocorre independentemente da viabilidade ou dormência das sementes, desde que não relacionada a impedimentos físicos à entrada de água. Os mesmos autores afirmam que a fase II é estacionária e ocorre em função do balanço entre o potencial osmótico e o potencial pressão. Nesta fase, a semente absorve água lentamente e o eixo embrionário ainda não consegue crescer. Na fase III ocorre novo aumento no grau de umidade das sementes e observa-se a emissão de raiz primária.

Deste modo, a importância da curva com as fases de entrada de água está relacionada tanto aos estudos de impermeabilidade de tegumento, como na determinação da duração de tratamentos com reguladores vegetais, condicionamento osmótico e pré-hidratação (Weaver, 1987; Carvalho \& Nakagawa, 2000; Albuquerque et al., 2000).

Diversos trabalhos avaliando a germinação de sementes de anonáceas têm sido realizados com reguladores vegetais, entre os quais Smet et al. (1999), com embebição de sementes de cherimóia em GA durante $24 \mathrm{~h}, 48 \mathrm{~h}$ e $72 \mathrm{~h}$ e Stenzel et al. (2003), com sementes de fruta-doconde em $\mathrm{GA}_{3}$ durante $24 \mathrm{~h}$; porém os autores não justificam fiosiologicamente os tempos empregados para os tratamentos.

Ferreira et al. (1997) observaram que sementes de Annona squamosa L. estabilizavam a entrada de água com aproximadamente 5 horas de imersão, caracterizando a mudança entre as fases I e II, enquanto, com 12 horas, as sementes de atemóia (Annona cherimola $\mathrm{x}$ A. squamosa) permaneciam na fase I. Por outro lado, o padrão trifásico de absorção de água foi observado em sementes de Solanum elaeagnifolium (Trione \& Cony, 1990), Glycine max (Armstrong \& Mcdonald, 1992), Cajanus cajan (Kalpana \& Rao, 1995) e Guazuma ulmifolia (Braga, 2003).

O objetivo deste trabalho foi caracterizar a curva de absorção de água em sementes de atemóia (Annona cherimola Mill x Annona squamosa L.) cv. Gefner com o emprego de diferentes métodos.

As sementes foram extraídas de frutos maduros adquiridos no município de Assai-PR, em pomar comercial de Edson Tomio Sato. O

\footnotetext{
${ }^{1}$ (Trabalho 075/2005). Recebido: 06/05/2005. Aceito para publicação: 08/02/2005.

${ }^{2}$ Prof $^{\mathrm{a}}$ Dr $^{\mathrm{a}}$ do Depto. de Botânica - IB/UNESP - Botucatu - SP, Rubião Jr.,s/n, CEP: 18618-000, C P: 510. E-mail: gisela@ibb.unesp.br.

${ }^{3}$ Prof. Dr. Centro de Ciências Agrárias, UNIOESTE - Câmpus de Mal Cândido Rondon - PR, R: Pernambuco, 1777, CEP: 85960-000.

${ }^{4}$ Prof $^{\mathrm{a}}$ Dr $^{\mathrm{a}}$ Depto. de Bioestatística - IB/UNESP - Botucatu - SP, Rubião Jr s/n, CEP: 18618-000, CP: 510.

${ }^{5}$ Mestrando em Agronomia, UNIOESTE - Câmpus de Marechal Cândido Rondon - PR, R: Pernambuco, 1777, CEP: 85960-000

${ }^{6}$ Doutorando em Ciências Biológicas - Botânica, IB/UNESP - Botucatu - SP, Rubião Jr s/n, CEP: 18618-000, CP..510.

${ }^{7}$ Graduanda em Agronomia, UNIOESTE - Câmpus de Marechal Cândido Rondon - PR, R: Pernambuco, 1777, CEP: 85960-000.
} 


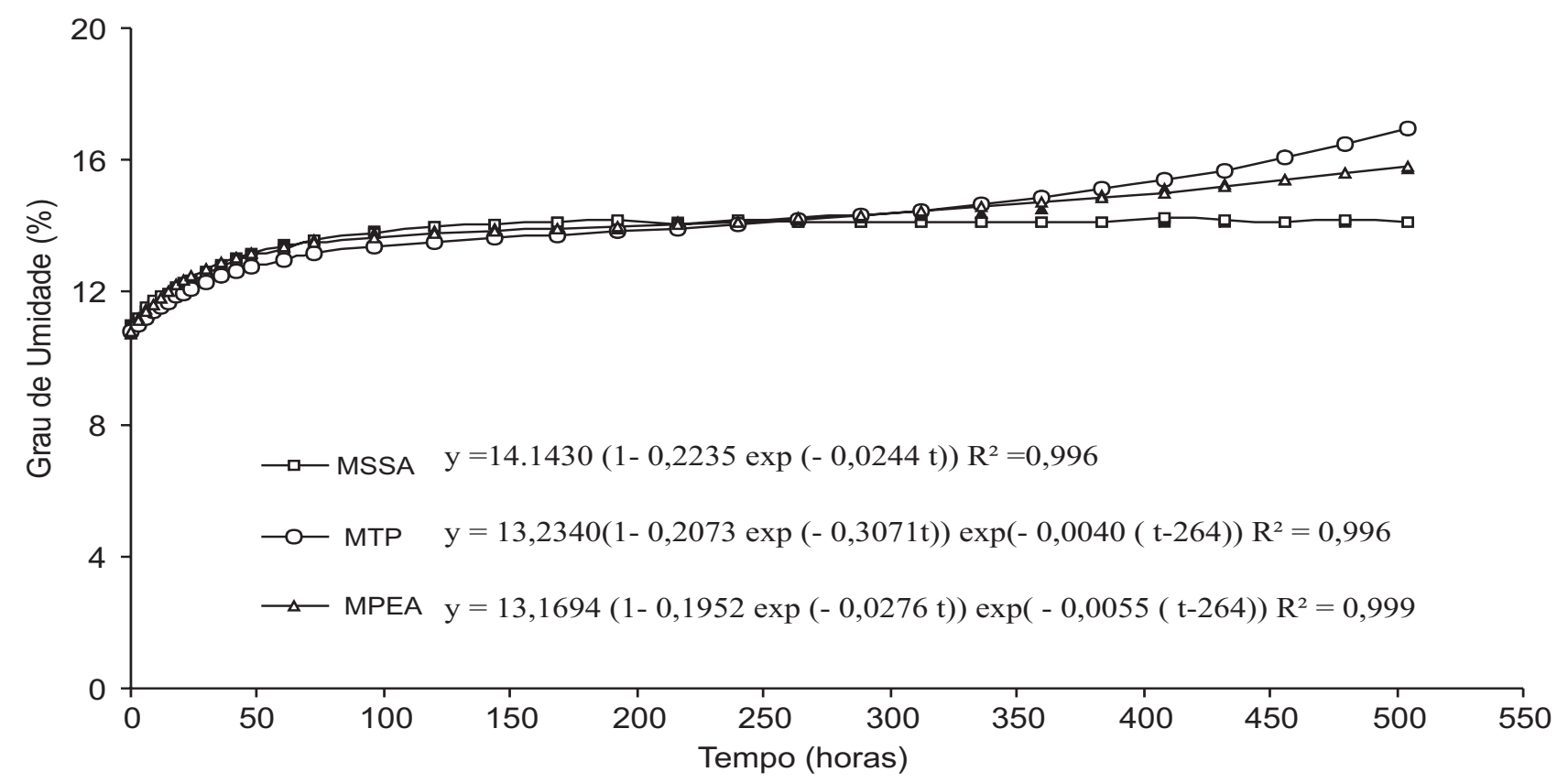

FIGURA 1 - Grau de umidade (\%) de sementes vivas de atemóia (A. cherimola Mill. x A. squamosa L.) cv. Gefner submetidas a três métodos de embebição: MSSA (sementes submersas em água destilada), MPEA (semente entre papel de filtro embebido em água destilada acondicionada em caixa tipo gerbox) e MTP (Teste-Padrão, com sementes mantidas em rolo de papel de filtro umedecido em água destilada). Mal. Cândido Rondon - PR, 2004.

delineamento experimental foi o inteiramente casualizado, com 6 tratamentos e 4 repetições de 25 sementes por parcela. Os tratamentos foram constituídos por três métodos de embebição, empregando-se sementes vivas e mortas. Para os tratamentos com as sementes mortas, realizou-se autoclavagem das sementes vivas por período de 20 minutos, temperatura de $121^{\circ} \mathrm{C}$ e pressão constante, e realizado teste de tetrazólio (Malavasi et al., 2001). Os métodos de embebição utilizados foram: papel embebido em água (MPEA), sementes submersas em água (MSSA) e teste-padrão (MTP). No método do papel embebido em água (MPEA), as sementes foram colocadas entre folhas de papel Germitest umedecido com água destilada, na quantidade de 2,5 vezes o peso do papel e acondicionadas em caixas gerbox. O método da semente submersa em água (MSSA) foi constituído pelo acondicionamento das sementes em béquer com $200 \mathrm{~mL}$ de água destilada e sistema de oxigenação. No método denominado teste-padrão (MTP), as sementes foram colocadas em rolo de papel Germitest umedecido com água destilada, empregando-se a quantidade de água referente a 2,5 vezes o peso do papel (Brasil, 1992).

Inicialmente, as sementes vivas e mortas foram pesadas, distribuídas nos tratamentos e acondicionadas em câmaras de germinação, com temperatura alternada de $20^{\circ} \mathrm{C} / 30^{\circ} \mathrm{C}(16 / 8$ horas $)$ e ausência de fotoperíodo. Após intervalos de tempo predeterminados (três horas no primeiro dia, seis horas no segundo, 12 horas no terceiro e a partir do quarto dia em intervalos de 24 horas até a observação da germinação visível, com emissão de $1 \mathrm{~mm}$ de raiz primária), as sementes eram retiradas dos tratamentos, secadas superficialmente com papel de filtro, pesadas, contadas as germinadas, colocadas novamente nos tratamentos e retornadas ao germinador, segundo método descrito por Baskin \& Baskin (2001).

A umidade inicial do lote foi obtida de acordo com Brasil (1992), e a cada pesagem, empregando-se os valores do peso total das sementes, calculava-se o teor de água em função do lote inicial (Menezes, 1996). A análise estatística foi realizada de acordo com Blacklow (1972), que considera a diferença de $1 \%$ entre o modelo estatístico ajustado e a sua assíntota, a fim de determinar os pontos de mudança entre as fases.

As Figuras 1 e 2 apresentam os resultados de absorção de água pelas sementes vivas e mortas de atemóia. Considerando o critério da diferença de $1 \%$ entre o modelo estatístico ajustado e a sua assíntota, identificaram-se os diferentes pontos de mudança da fase I para a II, em cada método, tanto para sementes vivas quanto para mortas. A curva de absorção que melhor representou as fases citadas por Bewley \& Black (1994) foi obtida empregando-se o MTP, com ponto de mudança entre as fases I e II após 27 horas de embebição e início da fase III após 234 horas (Figura 1). Embora Carvalho \& Nakagawa (2000) relatem que a fase I seja relativamente rápida, com duração de uma a duas horas, neste trabalho, foram necessárias 27; 34 e 47 horas para os métodos MTP, MPEA e MSSA, para diminuir a absorção rápida de água (embebição) e entrar na fase II, comportamento que foi observado tanto para sementes vivas (Figura 1) como para mortas (Figura 2).

O tempo da fase I está de acordo com citações de Coll et al. (2001) de que a velocidade de absorção e a quantidade de água embebida variam com a natureza e composição do tegumento, fato comprovado também por Ono et al. (1993) com sementes de macadâmia, Rossetto et al. (1997) com sementes de soja, Braga (2003) com sementes de Guazuma ulmifolia e Ferreira et al. (1997) com sementes de fruta-do-conde e atemóia, com tempo de duração da fase I de $90 \mathrm{~h}, 12 \mathrm{~h}, 6 \mathrm{~h}, 5 \mathrm{~h}$, respectivamente, exceto as sementes de atemóia que, com $12 \mathrm{~h}$, continuavam embebendo.

A permanência na fase II foi semelhante para todos os tratamentos com sementes vivas e mortas, porém a fase III foi observada somente nos tratamentos MTP e MPEA com aproximadamente 234 horas após o início da embebição (Figura 1), o que se torna mais visível observando-se conjuntamente a Figura 2, na qual as sementes mortas permanecem indefinidamente na fase II.

A embebição de sementes de anonáceas por períodos de 24 horas em água, conforme sugerem Garner \& Chaudri (1976), ou por 48 horas, conforme Lemos et al. (1988), não pode ser generalizada para as diversas espécies de Anonaceae, uma vez que, para atemóia, cv. Gefner, esse período varia de 27 a 47 horas de embebição, dependendo do método empregado.

Os métodos que caracterizaram as três fases da curva de absorção de água em sementes de atemóia (Annona cherimola Mill. x A. squamosa L.) cv. Gefner foram o MTP e MPEA, cuja mudança da fase I para a II foi de 27 e 34 horas, respectivamente, atingindo a fase III com 234 horas, o que permite determinar o tempo de embebição para tratamentos pré-germinativos. 


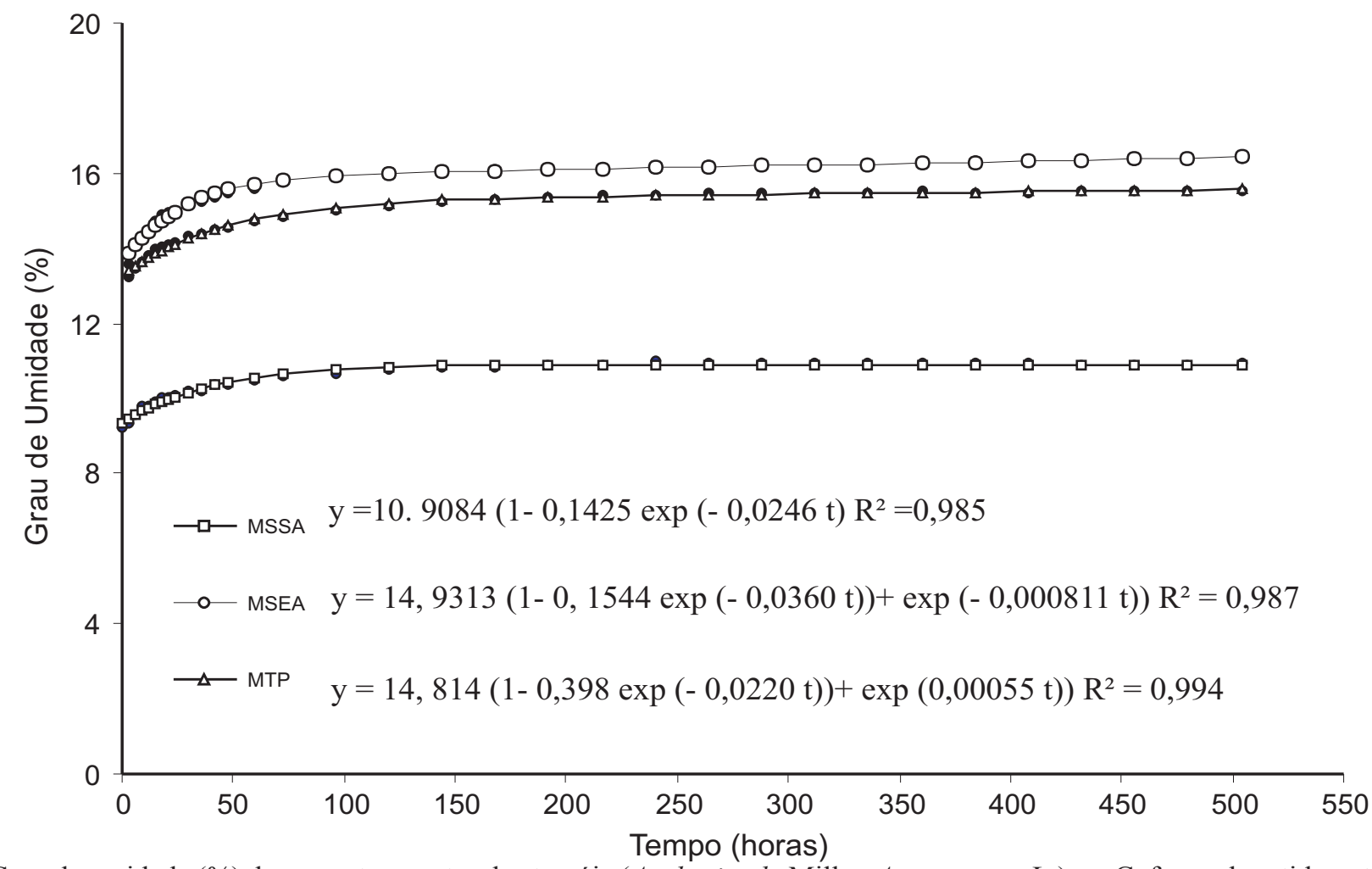

FIGURA 2 - Grau de umidade (\%) de sementes mortas de atemóia (A. cherimola Mill. x A. squamosa L.) cv. Gefner submetidas a três métodos de embebição: MSSA (sementes submersas em água destilada), MPEA (semente entre papel de filtro embebido em água destilada acondicionada em caixa tipo gerbox) e MTP (Teste-Padrão, com sementes mantidas em rolo de papel de filtro umedecido em água destilada). Mal. Cândido Rondon - PR, 2004.

\section{REFERÊNCIAS}

ALBUQUERQUE, M.C.F.; RODRIGUES, T. de J.D.; MENDONÇA, E.A.F. Absorção de água por sementes de Crotalaria spectabilis Roth determinada em diferentes temperaturas e disponibilidade hídrica. Revista Brasileira de Sementes, Brasília, v. 22, p. 206-215, 2000.

ARMSTRONG, H.; McDONALD, M.B. Effects of osmo-conditioning on water uptake and electrical conductive in soybean seeds. Seed Science \& Technology, Zürich, v.20, n.3, p.391-400, 1992.

BASKIN, C.C.; BASKIN, J.M. Seeds: ecology, biogeography, and evolution of dormancy and germination. New York: Academic Press, 2001.666p.

BEWLEY, J.D.; BLACK, M. Seeds: physiology of development and germination. $2^{\text {nd }}$ ed. New York: Plenum Press, 1994. 445p.

BLACKLOW, W.M. Mathematical description of the influence of temperature and seed quality on the imbibition by seeds of corn (Zea mays L.). Crop Science, Madison, v. 12, p. 643 - 646, 1972.

BOURKE, D. O’D. Annona ssp. In: GARNER, R. J.; CHAUDHRI, S. A. The propagation of tropical fruit tree. Maidstone: FAO/ CAB, 1985. p. $223-247$.

BRADFORD, K.J. Water relations analysis of seed germination. In: KIGEL, J.; GALILI, G. (Ed.). Seed development and germination. New York: Marcel Decker., 1995. p. 351 - 396.

BRAGA. J.F. Germinação de sementes de mutamba (Guazuma ulmifolia Lam. - Sterculiacea): dormência, temperatura, fotoblástismo e potencial osmótico. 2003. 61f. Dissertação (Mestrado) - Instituto de Biociências, Universidade Estadual Paulista, Botucatu, 2003.

BRASIL. Ministério da Agricultura e Reforma Agrária. Regras para análise de sementes. Brasília: SNDA/DNDV/CLAV, 1992.365p.

CARVALHO, N.M.; NAKAGAWA, J. Sementes: ciência, tecnologia e produção. 4. ed. Jaboticabal: FUNEP, 2000. 588p.

CEREDA, E.; FERREIRA, G. Propagação vegetativa de anonáceas por estaquia. In: SÃO JOSÉ, A.R.; SOUZA, I.V.B.; MORAIS, O.M.; REBOLÇAS, T.N.H. Anonáceas, produção e mercado (pinha, graviola, atemóia e cherimólia). Vitória da Conquista, BA: DZF/ EUSB, 1997.p. $257-262$.
COLL, J.B.; RODRIGO, G.N.; GARCIA, B.S.; TAMES, R.S. Fisiologia vegetal. Madrid: Ediciones Pirámide, 2001.566p.

EIRA, M.T.S.; CALDAS, L.S. Seed dormancy and germination as concurrent processes. Brazilian Journal of Plant Physiology, Piracicaba, v. 12, p. 85-103, 2000.

FERREIRA, G.; CEREDA, E.; SILVA, C.P.; CUNHA, R.J.P; CATANEO, A. Imbibition study of sugar apple (Annona squamosa L.) and atemoya (Annona cherimola Mill. e Annona squamosa L.). Seeds. In: CONGRESSO INTERNACIONAL DE ANONÁCEAS, 1997, Chapingo, Mexico. Memórias.... Chapingo: Universidad Autonoma Chapingo, 1997. p. 210-224.

GARNER, R.J.; CHAUDRI, S.A. The propagation of tropical fruit trees. East Malling: Commenwlth Bureau of horticulture and Plantation Crops, 1976. 566p. (Horticultural Review, 4).

KALPANA, R.; RAO, K.V.M. on the ageing machanism in pigeonpea (Cajanus cajan (L.) Millsp.) seeds. Seed Science \& Technology, Zürich, v.23, n. 1, p. 1-9, 1995.

KAVATI, R.O.; BUENO, S.C.S.; TOKUNAGA, T.; NOGUEIRA, E.M.C.; TAKASSAKI, J.; PERIOT, N.W. A cultura da atemóia (Annona cherimola Mill. x Annona squamosa L.) Campinas: CATI, Departamento de Comunicação e Treinamento, 1997. 22p.

LABORIAU, L. G. A. Germinação das sementes. Washington: Secretaria Geral da Organização dos Estados Americanos, 1983. 171p.

LEMOS, E.E.P.; CAVALCANTI, R.L.R.R.; CARRAZONI,A.A.; LOBATO, T.M.L. Germinação de sementes de pinha submetidas a tratamentos para quebra de dormência. In: CONGRESSO BRASILEIRO DE FRUTICULTURA, 9., 1988, Campinas. Anais... Campinas: Sociedade Brasileira Fruticultura, 1988. v.2, p. $675-678$

MALAVASI, M. M.; FOGAÇA, C. A.; FOGAÇA, L.; FERREIRA, G. Preparo e coloração de sementes de maracujá-doce (Passiflora alata Dryander) para a avaliação da viabilidade através de teste do tretazólio. Revista Brasileira de Fruticultura, Campinas, v.23, n.1, p.126-129, 2001.

MENEZES, D. Determinação da curva de embebição e avaliação da quantidade fisiológica de sementes de algodão (Grossypium hirsutur L.). 1996. 73f. Dissertação (Mestrado em Fitotecnia)- Universidade 
Federal de Lavras, Lavras, 1996.

ONO, E. O.; RODRIGUES, J. D.; SABINO, J. C.; de PINHO, S. Z. Estudo da embebição e da viabilidade de sementes de macadâmia (Macadamia integrifólia MAIDEN \& BETCHE). Sciencia Agrícola, Piracicaba, v.50, n. 1, p. 40-44, 1993.

PAWSHE, Y.H.; PATIL, B.N.;PATIL, L.P. Effect of pregermination seed tratment on the germination and vigour of seedlings in custard apple (Anona squamosa L.). Annals of Plant Physiology, v.11, n.2, p. $150-154,1997$.

ROSSETO, C.A.V.; NOVEMBRE, A.D da A.L.C.; MARCAS FILHO, J.; DA SILVA, W.R.; NAKAGAWA, J. Efeito da disponibilidade hídrica do substrato, da qualidade fisiológica e do teor de água inicial das sementes de soja no processo de germinação. Scientia Agricola, Piracicaba, v. 54, n. 1-2, p. $97-105,1997$.

SAMPAIO, V.R. Propagação das frutas tropicais. In: DONADIO, L.C. (Ed.). Fruticultura tropical. Jaboticabal: FUNRP, 1992.p. $233-245$.

SANEWSKI, G.M. Custard apples: cultivation and crop protection. Brisbane: Queensland Departament of Primary Industries, 1991. 103p.
SMET, S.DE.; DAMME, P. VAN.; SCHELDEMAN, X.; ROMERO, J. Seed structure and germination of cherimoya (Anona cherimola Mill.). Acta Horticulturae, Wageningen, n. 497, p. 269-278, 1999.

STENZEL, N.M.C. A cultura da atemóia no Estado do Paraná. In: SÃO JOSÉ, A.R.; SOUZA, I.V.B.; MORAIS, O.M.; REBOLÇAS, T.N.H. Anonáceas, produção e mercado (pinha, graviola, atemóia e cherimólia). Vitória da Conquista, BA: DFZ/ UESB, 1997. p. 307 308.

STENZEL, N.M.C.; MURATA, I. M.; NEVES, C. S. V. J. Superação da dormência em sementes de atemóia e fruta-do-conde, Revista Brasileira de Fruticultura, Jaboticabal, v.25, n. 2, p.305-308, 2003.

TRIONE, S.; CONY, M. A. Thermoperioclism and other physiological traits of solanum elaeagnifolium seeds in relation to germination. Seed Science \& Technology, Zürich, v. 18, n.1, p. 25-39, 1990.

WEAVER, R. J. Reguladores del crescimento de las plantas em la agricultura. $5^{\text {th }}$ ed. México: Trillas, 1987.622p.(Footnotes) 